\title{
CARACTERÍSTICAS EXPERIMENTAIS DAS PUBLICAÇÕES DA CIÊNCIA RURAL DE 1971 A 2000
}

\author{
CHARACTERISTICS OF THE PUBLICATIONS IN THE CIÊNCIA RURAL FROM 1971 TO 2000
}

\author{
Alessandro Dal'Col Lúcio ${ }^{1}$ Sidinei José Lopes ${ }^{2}$ Lindolfo Storck $^{3}$ \\ Ricardo Howes Carpes $^{4}$ Daniel Lieberknecht $^{5}$ Márcia Cristina Nicola $^{6}$
}

\author{
- NOTA - \\ RESUMO

\section{ABSTRACT}

Com o objetivo de caracterizar os trabalhos publicados na Ciência Rural entre 1971 e 2000 e verificar adequação ou não do emprego de análises estatísticas, foram catalogados 1788 trabalhos, sendo 860 da área animal e 928 da vegetal. Dentro de cada área, foram obtidos a freqüência relativa simples das estruturas experimentais e dos testes estatísticos de análise complementar aplicados para tratamentos qualitativos e quantitativos e os valores máximos, mínimos e médios para números de tratamentos, de repetições e para o nível de erro. Na área animal, a predominância dos trabalhos foi utilizar 3 tratamentos, 4 repetições, nível de 5\% de probabilidade de erro, teste de Tukey para comparar tratamentos qualitativos e análise de regressão linear simples para tratamentos quantitativos. Na área vegetal, a predominância foi usar o delineamento em blocos ao acaso, com 6 tratamentos, 5 repetições, nível de $5 \%$ de probabilidade de erro, teste de Duncan para tratamentos qualitativos e análise de regressão linear simples para tratamentos quantitativos. Na classificação da adequação dos testes de análise complementar, na área animal foi de Adequado $=63 \%$ e Inadequado $=26 \%, e$ na área vegetal de Adequado $=61 \%$ e Inadequado $=25 \%$ que mostrou que a maioria dos trabalhos possuem adequada utilização dos testes nas análises complementares nos trabalhos deste periódico.

Palavra-chave: Análise de regressão; teste de comparação de médias; planejamento experimental; análises estatísticas.
With the objective of describing the characteristics of the works published in the Ciência Rural between 1971 and 2000 and to identify deficiencies in the employment of statistical analysis, 1788 works were classified, being 879 of the animal area and 909 of the vegetable. Inside of each area it was obtained the simple relative frequency of the experimental desing and of the statistical tests of complemental analysis applied for qualitative and quantitative treatments and, the maximum values, minima and medium for numbers of treatments and repetitions and for the level of used error, showing that in the animal area, the profile of the works is of using 3 treatments, 4 repetitions, level of 5\% of probability error, test of Tukey for qualitative treatments and analysis of simple lineal regression for quantitative treatments. Already in the vegetable area, the profile was maybe of using the delineamento in blocks to the, with 6 treatments, 5 repetitions, level of 5\% of probability error, test of Duncan for qualitative treatments and analysis of simple lineal regression for quantitative treatments. In the classification of the adaptation of the tests of complemental analysis, in the animal area it was Appropriate $=63 \%$, Inadequate $=26 \%$, and vegetable of Approppriate $=61 \%$, Inadequate $=25 \%$, showing that most of the works possesses appropriate use of the tests in the complemental analyses, could give a good reliability to the results and conclusions obtained in these works.

Key words: Regression analysis; averages comparison test; experimental planning; statistical analysis.

${ }^{1}$ Engenheiro Agrônomo, Doutor., Professor Adjunto Departamento Fitotecnia, Centro de Ciências Rurais (CCR), Universidade Federal de Santa Maria (UFSM), 97105-900, Santa Maria - RS. E-mail: adlucio@ccr.ufsm.br. Autor para correspondência.

${ }^{2}$ Engenheiro Agrônomo, MSc., Prof. Assistente Departamento Fitotecnia, CCR, UFSM, Doutorando ESALQ, USP, 97105-900, Santa Maria - RS. E-mail: sjlopes@ccr.ufsm.br.

${ }^{3}$ Engenheiro Agrônomo, Doutor, Professor Titular Departamento Fitotecnia, CCR, UFSM, 97105-900. Santa Maria - RS. Bolsista CNPq. E-mail: storck@ccr.ufsm.br.

${ }^{4}$ Acadêmico do Curso de Graduação em Agronomia, CCR, UFSM. Bolsista FIPE, UFSM.

${ }^{5}$ Acadêmico do Curso de Graduação em Agronomia, CCR, UFSM. Bolsista FAPERGS.

${ }^{6}$ Engenheiro Agrônomo, MSc. em Produção Vegetal, Universidade Federal de Pelotas. 


\section{INTRODUÇÃO}

A Revista Ciência Rural foi criada em 1971, com o nome de Revista do Centro de Ciências Rurais e, desde então, tem sido um espaço para a publicação dos trabalhos realizados pelo corpo docente do Centro de Ciências Rurais da Universidade Federal de Santa Maria. Atualmente é uma das revistas científicas em agropecuária mais importantes do Brasil, recebendo e publicando também artigos e relatos de trabalhos e pesquisas realizados em diversas regiões do país e do mundo. As publicações de trabalhos externos à UFSM aumentam a cada ano. Em 1991, foram em torno de 7\% os trabalhos publicados, em 2000, foram aproximadamente $70 \%$.

$\mathrm{Na}$ pesquisa agropecuária, os dados obtidos nos experimentos, quando atendem às pressuposições do modelo matemático, são passíveis de serem submetidos a algum tipo de análise estatística, sendo a mais freqüente a análise da variância, na qual, segundo BARBIN (1993), é importante ter conhecimento da natureza dos tratamentos avaliados, pois se estes são de efeito fixo, a análise aplicada visa estimar os efeitos individuais de cada um e compará-los entre si e, quando os tratamentos são de efeito aleatório, a análise visa à estimação dos componentes da variância, que possuem grande importância no melhoramento genético, animal ou vegetal.

Na comparação das estimativas dos tratamentos de efeito fixo, são empregados testes conhecidos como Testes de Comparação Múltipla de Médias (TCMM), quando estes tratamentos ou fatores possuem características qualitativas e, Análise de Regressão (AR) para diferentes modelos quando os tratamentos são quantitativos (BANZATTO \& KRONKA, 1995; STORCK et al. 2000; GOMES, 2000). De acordo com CARDELLINO \& SIEWERDT (1992), esses procedimentos estatísticos são freqüentemente empregados de forma indiscriminada, prejudicando as conclusões obtidas a partir dos resultados apresentados, pois deixa-se de obter informações sobre tratamentos intermediários, como contrastes economicamente significativos e pontos de máxima eficiência técnica e econômica.

O presente trabalho teve por objetivos descrever as características experimentais dos trabalhos publicados na Ciência Rural entre 1971 e 2000 e identificar possíveis falhas no emprego de análises estatísticas.

Foram catalogados um total de 1788 trabalhos publicados na Ciência Rural, com 879 da área animal e 909 da vegetal, entre os números 01 de 1971 e 06 de 2000, sendo artigos científicos, nota científica, relato de caso e revisão bibliográfica. Os artigos e notas for- maram um grupo (AN) e outro grupo foi composto pelos relatos de caso e revisões bibliográficas (RR).

Para cada trabalho, foram anotadas as seguintes informações: a) tipo de trabalho (AN ou RR); b) área da ciência agropecuária: animal ou vegetal; c) estrutura experimental aplicada; d) número de tratamentos; e) número de repetições; f) tipo de teste qualitativo aplicado; g) tipo de teste quantitativo aplicado e; h) nível de erro utilizado. Foi caracterizada a metodologia experimental dos trabalhos do grupo AN, dentro de cada área específica, obtendo-se as freqüências relativas simples dos tipos de estrutura utilizados e tipos de testes qualitativos e quantitativos aplicados. Ainda foram obtidos os números mínimo, máximo e médio para número de tratamentos e de repetições e nível de erro. Com os trabalhos, do tipo AN, publicados desde o número 01 de 1990 até o 06 de 2000, dentro de cada área, foi verificada a adequação ou não dos testes qualitativos e quantitativos aplicados, a partir do critério descrito por SANTOS et al. (1998), classificando-os em uso adequado, inadequado, parcialmente adequado e não identificado. O parcialmente adequado foi atribuído quando se tratava de fatorial e um dos testes foi adequado e outro não e/ou quando os dados poderiam ser melhor explorados estatisticamente.

Na tabela 1, observa-se que o número de repetições e do nível de erro, foram semelhantes para as áreas animal e vegetal, mostrando uma coerência entre estas, no planejamento experimental dos trabalhos. Estes valores comprovam que, independente da área da ciência agropecuária, existe uma estrutura padrão de experimento, com poucos tratamentos, 4 repetições e um nível de erro de 5\%. Sem considerar o delineamento experimental utilizado, constatou-se que à medida que o número de tratamentos reduzia, o número de repetições aumentava, mantendo o grau de liberdade do erro (gle) em níveis aceitáveis.

Na escolha do delineamento experimental, há uma situação inversa, quando se compara as duas áreas, porque na área animal há preferência para a utilização de amostragem, seguido do delineamento inteiramente casualizado (DIC) e do blocos ao acaso (DBC). Já a freqüência de uso do DIC é devido à homogeneidade das unidades experimentais, mais facilmente pressuposta na área animal, mantendo condições de ambiente e de manejo homogêneos durante todo o experimento. Na área vegetal, há uma semelhança entre o emprego da amostragem e do DBC, seguidos do DIC, mostrando que, nessa área, pelo fato de a maioria dos trabalhos serem conduzidos a campo, normalmente a heterogeneidade do solo exige que seja utilizado o bloqueamento desta fonte de heterogeneidade. 
Tabela 1. Valores mínimo, máximo e médio, para número de tratamentos (I) e repetições (J) e do nível de erro ( $\alpha \%)$, freqüências relativas simples $(\%)$ das estruturas experimentais e dos testes estatísticos de análise complementar utilizados nos trabalhos publicados na Revista Ciência Rural, entre os anos de 1971 e 2000 e classificação dos trabalhos, em percentagem, das áreas animal e vegetal, publicados entre os anos de 1990 e 2000, conforme adequação da aplicação dos testes estatísticos para análise complementar. Santa Maria - RS, 2001

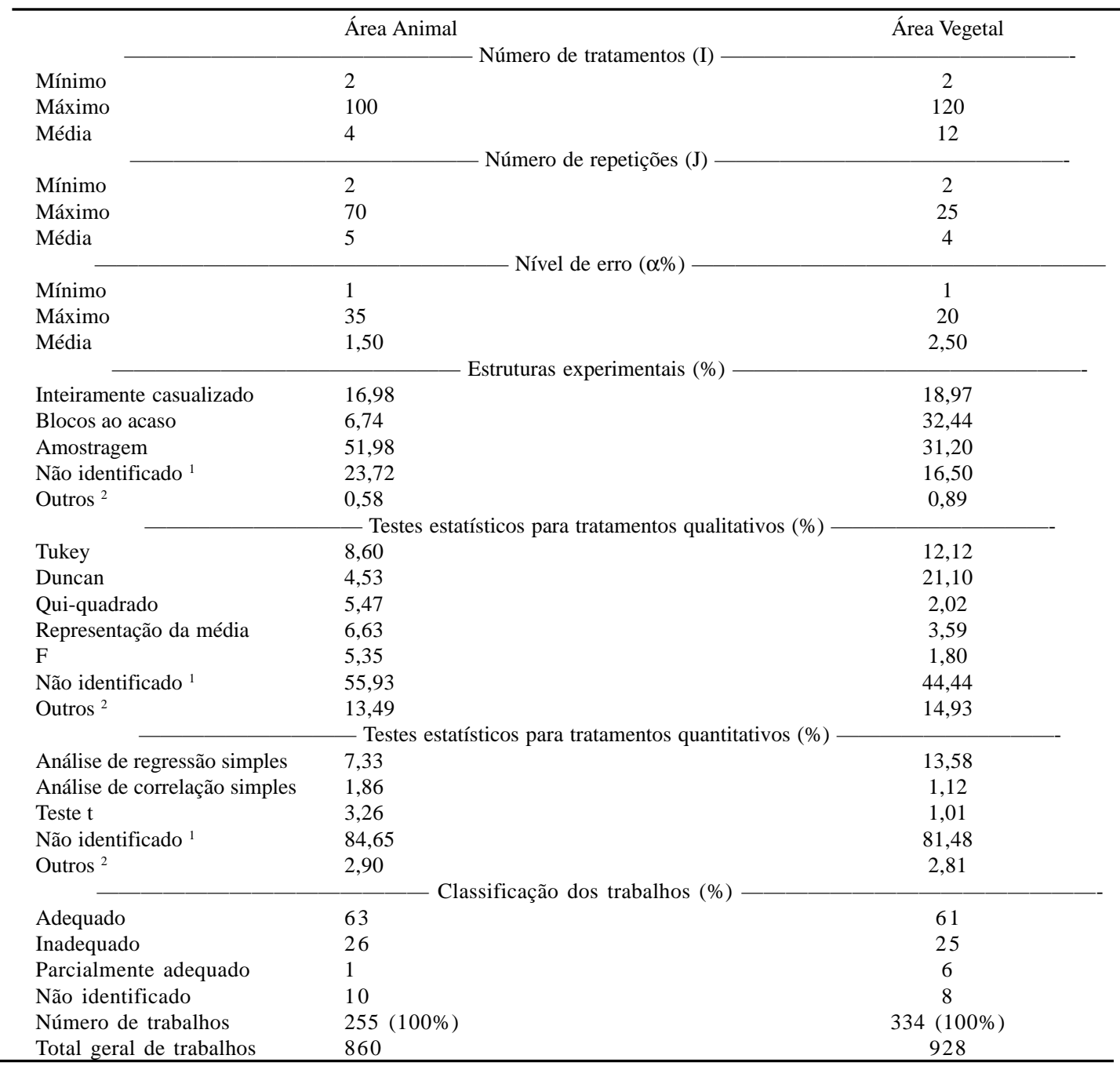

${ }^{1}$ : Trabalhos nos quais as informações sobre delineamento experimental e teste estatístico para análise complementar não foram relatadas ou não foram aplicados, como nas revisões bibliográficas e relatos de caso;

2 : Estruturas experimentais ou testes estatísticos nos quais a freqüência relativa simples individual, foi inferior a $1 \%$.

No uso de testes para comparação de tratamentos qualitativos, independente da área, há o mesmo comportamento de quais dos testes empregados, apesar de freqüências diferentes. Ao se verificar a freqüência de uso destes testes em relação ao número de tratamentos, observou-se que os testes de Tukey e Duncan foram utilizados quando o número de tratamentos foi menor que 20, na maioria dos casos e, quando este aumentava, deu-se preferência ao uso dos testes Qui-quadrado $\left(\chi^{2}\right)$ e de F e a simples representação das médias de cada tratamento com seus respectivos desvios. Provavelmente este resultado foi obtido pela falta de informação ou desconhecimento dos pes- quisadores responsáveis pelos trabalhos, pois a opção de não aplicar um teste de comparação de médias, quando o número de tratamentos é alto, não procede, sendo recomendado nestes casos a aplicação do teste SCOTT \& KNOT (1974), que possui a mesma precisão que o Duncan, apresentando um menor número de grupos de tratamentos semelhantes. Já na área vegetal, deu-se preferência ao teste de Duncan, que possui como característica uma maior discriminação dos tratamentos quando comparado com o teste de Tukey.

O comportamento das áreas animal e vegetal foi semelhante quando analisado em relação aos testes estatísticos para análise complementar de tratamentos 
quantitativos, sendo verificado que as análises de regressão linear simples e de correlação foram aplicadas quando o número de tratamentos foi inferior a $25 \mathrm{e}$, quando estes foram altos, foi usado o teste t (Tabela 1). A opção de aplicar a análise de regressão linear simples foi maior na área vegetal, mas isto não indica que os trabalhos da área animal, onde os tratamentos foram quantitativos e foi aplicado o teste t, estão errados. Esta opção na área animal deu-se, muitas vezes, pelo motivo de os animais serem avaliados no tempo, que é um fator quantitativo passível de análise de regressão, e nem todos os tempos de análise serem interessante na prática, sendo então aplicado o teste t para comparação dos tratamentos de interesse prático ou econômico.

Na classificação da adequação dos testes aplicados nas análises complementares dos experimentos, independente desses serem com tratamentos qualitativos ou quantitativos, quando os trabalhos tanto da área animal quanto da vegetal, apresentaram um bom resultado, (em mais de 60\% dos trabalhos), os autores utilizaram de forma adequada os testes, mostrando uma boa qualidade e confiabilidade nos resultados e conclusões apresentadas por estes trabalhos. Esses resultados são contrários aos obtidos por CARDELLINO \& SIEWERDT (1992) e SANTOS et al (1998), que avaliaram como inadequada, a maioria dos trabalhos analisados por testes de comparação múltipla de médias, respectivamente na Revista da Sociedade Brasileira de Zootecnia e da Pesquisa Agropecuária Brasileira.

Cabe salientar que a percentagem de trabalhos com uso inadequado é alto, 26 e $25 \%$ respectivamente para as áreas animal e vegetal, pois com as facilidades computacionais existentes, no que se trata de software estatísticos disponíveis, e maior difusão das técnicas de planejamento e análise de experimentos, como, por exemplo, artigos e textos de DRAPER
\& SMITH (1966), PERECIN \& MALHEIROS (1989), BANZATTO \& KRONKA (1995), GOMES (2000) e STORCK et al. (2000), os dados provenientes de experimentos têm condições suficientes de serem analisados adequadamente, dando maior confiabilidade aos resultados e conclusões obtidas. Ainda, a não indicação do teste aplicado pode ser um indício de má qualidade das análises, descaso ou desconhecimento de experimentação por parte do pesquisador, pois como já mencionado, a difusão das técnicas experimentais dá condições de, pelo menos, saber descrever o que foi realizado no trabalho.

\section{REFERÊNCIAS BIBLIOGRÁFICAS}

BANZATTO, D.A.; KRONKA, S.N. Experimentação agrícola. 3.ed. Jaboticabal : FUNEP, 1995. 247p.

BARBIN, D. Componentes de variância: teoria e aplicações. 2.ed. Piracicaba : FEALQ, 1993. 120p.

CARDELLINO, R.A.; SIEWERDT, F. Utilização adequada e inadequada dos testes de comparação de médias. Revista da Sociedade Brasileira de Zootecnia, Viçosa, v.21, n.6, p.985995, 1992.

DRAPER, N.R; SMITH, R. Applied regression analysis. Nova York : John Wiley, 1966. 407p.

GOMES, F.P. Curso de estatística experimental. 15.ed. Piracicaba : Nobel, 2000. 468p.

PERECIN, D.; MALHEIROS, E.B. Procedimentos para comparações múltiplas. Lavras : UFLA, 1989. 67p.

SANTOS, J.W.; MOREIRA, J.A.N.; BELTRÃO, N.E.M. Avaliação do emprego dos testes de comparação de médias na revista Pesquisa Agropecuária Brasileira (PAB) de 1980 a 1994. Pesquisa Agropecuária Brasileira, Brasília, v.33, n.3, p.225$230,1998$.

SCOTT, A.J.; KNOTT, M. A cluster analysis methods for grouping means in the analysis of variance. Biometrics, Raleigh, v.30, n.3, p.507-512, 1974.

STORCK, L. et al. Experimentação vegetal. Santa Maria : UFSM, 2000. 198p. 